\title{
A new generalized meshless method technique for tracheobronchial airflow modeling
}

\author{
K. Erhart ${ }^{1}$, E. Divo ${ }^{1}$, A. Kassab ${ }^{1}$, S. Gerace ${ }^{1}$, A. Santhanam ${ }^{1,2}$ \\ \& P. Kupelian ${ }^{2}$ \\ ${ }^{1}$ University of Central Florida, USA \\ ${ }^{2}$ M. D. Anderson Cancer Center, USA
}

\begin{abstract}
Numerous approaches to the implementation of Meshless methods have been developed and described over the last several years for a wide range of physical applications. Despite this wide range of applications and implementation approaches, few attempts have been made to combine the various schemes utilized throughout the Meshless research community in the hopes of achieving a more resilient and accurate Meshless method. This paper describes such a generalized Meshless approach that incorporates moving least squares, radial basis functions, and finite differencing into a unique Meshless-based solution framework. The development of this approach was stimulated by stability requirements that arise in the difficult geometries that appear in typical biological flow modeling applications. Therefore, this new approach is described in the context of tracheobronchial flow modeling for the prediction of thoracic tumor movement throughout the breathing cycle.
\end{abstract}

Keywords: meshless methods, generalized finite differencing, porous lung flow.

\section{Introduction}

The medical community is currently giving much attention to the idea of individualized medical treatment. Individualized treatment means that medication, surgery, and other procedures are selected based on the specific details of each patient, rather than through a more general disease based protocol. This process allows the use of drugs and treatments that may be harmful to some patients to be utilized successfully on those patients that have conditions and circumstances ideally suited to the treatment. The area of cancer treatment has been following 
this trend for some time, as size and anatomy can vary significantly from patient to patient. In conformal and intensity modulated radiation therapy for example, the exact details of the individual patient anatomy are absolutely critical to successful treatment outcomes. This is especially important for cancers in anatomic regions that experience motion due to breathing, such as the lungs, chest, and abdomen. Therefore much effort has recently gone into the area of real-time (4D) medical imaging. These advanced imaging techniques have made it possible to quantify expected tumor motions throughout the breathing cycle and have provided confidence that radiation is being delivered to the correct cancer site. Despite these improved imaging capabilities, there is currently no process available to visualize tumor motion during the actual course of radiation treatment. Therefore, the medical staff must rely upon previously obtained images to predict the motion during the actual treatment procedure. This research aims to improve these prediction capabilities by correlating real-time airflow data with the lung/tumor motion. A standard spirometer can be used to monitor the patient's breathing rate and computational fluid dynamics and mechanics data are used to predict the resulting lung deformations, thereby providing an improved means of estimating the current tumor position. In addition, the imaging data and computational models can be used to improve the accuracy of lung tissue property estimations so that patient treatment and tissue complications can be more accurately assessed.

This paper will introduce a new generalized Meshless computational modeling approach, describe its application to the area of tracheobronchial flow modeling, and discuss the preliminary findings of the research. The computational modeling has been carried out using a Meshless-based approach for both flow and elasticity analysis. Meshless methods offer an extremely increased ease of use for biological applications as individual patient anatomies are quite complex and therefore very difficult to represent with typical finite element or finite volume meshes. However, as with any automatically discretized technique, solution stability and robustness are extremely key factors to the methodology and therefore have been key aspects in guiding this research.

In order to present this research in the most suitable fashion, this paper is organized into several sections, which are then followed by some overall concluding remarks. In the first section, we will present a more complete description of the research goals, tasks, and approach. This section will describe the entire solution procedure and coupling process as it is not tied specifically to Meshless methods, therefore the ideas and approaches utilized within this work can be understood by those without experience in the field of Meshless method modeling. Following this application overview, a section describing the pertinent details of our new generalized Meshless technique will be presented along with numerous references to more detailed Meshless method publications. Finally, results will be presented to demonstrate the current progress that has been made in individualized patient tracheobronchial airflow modeling for cancer treatment applications. 


\section{Tracheobronchial airflow modeling}

Despite large reductions in smoking habits and decreased use of harmful building materials and chemicals, lung cancer is still the second most common type of cancer in the United States. While radiation therapy has made huge strides in offering curative treatments for many types of cancer, its use in treating lung cancer is particularly difficult due to motion of the tumor that is induced by normal patient breathing. Due to the length of the treatment and the relative weakness of many of the lung cancer patients, simply restricting or reducing breathing during treatment is not a viable means of reducing tumor motion (radiation treatment is typically delivered in 5-15 minute doses over multiple consecutive days). Additionally, the motion patterns of many lung tumors can vary unpredictably from day-today $[1,2]$, the depth of breathing can change even in short amounts of time, and there are notable individual differences in breathing and motion patterns from one patient to another. These difficulties complicate treatment and since providing adequate radiation dose to the tumor is the primary goal of radiation therapy, treatment fields are often enlarged to accommodate for these motions and uncertainties. This results in higher doses to normal tissue, thereby increasing the toxicity rates from therapy. Through advanced medical imaging it has been found that lung tumors can move as much as $3 \mathrm{~cm}$ while the average tumor size in patients is only $5.5 \pm 3.1 \mathrm{~cm}$ [1], therefore this practice of field enlargement can easily lead to over 50\% increases in the field size, much of which would be unnecessary if a more confidence could be assured in the precise tumor position and motion patterns. It is precisely the goal of this research to address this need.

The goal of this research, improving tumor motion predictions, has been used to guide the development of an appropriate physical model of the tracheobronchial system. Keeping this overall goal in mind has allowed for several assumptions to be made regarding the model physics, without any significant depreciation of overall motion prediction capabilities. First, the oral cavity and mouth have been removed from the flow models and the system inlet begins directly after the larynx. This assumption is justified by noting that the oral cavity flow cross sections are much larger than those within the trachea, therefore the pressure drop from the mouth to the start of the trachea will be insignificant when compared to the overall flow through the trachea. Our second physical simplification is to model the lung as a continuous porous medium with variable, patient specific, material properties. This approach has been used with success by previous investors for airflow modeling within lungs [3]. Our third and final simplification involves both physics and geometric assumptions. Since the trachea branches into smaller passageways numerous times before and within the lung, some decision must be made regrading where to separate the trachea from the lung itself. There are several trade-offs that must be investigated when making this decision, since as more levels are included: the flow passageways become increasing small so that eventually the continuum flow model assumptions will break down, the number of interfaces between the lung and trachea models increases, extracting the passageways from the medical images becomes more difficult, and consistency between the model and real 
patient is improved. We have found that the positive effect of increasing the number of branching levels on consistency is quickly degraded by the loss of geometric accuracy in obtaining the flow geometry from the medical images. Therefore, we have limited the branching to only the first two levels, as shown schematically in Figure 1.
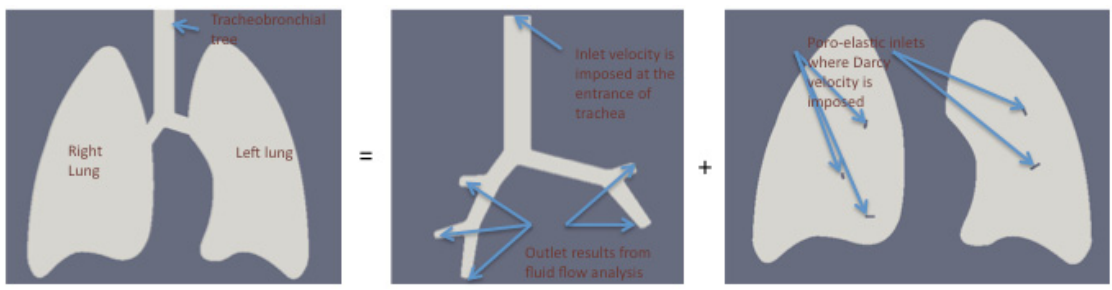

Figure 1: Coupling of the trachea and lung models.

Now that the major assumptions have been stated, we can begin to detail the overall process of modeling the tracheobronchial flow system. Mass flow rate and pressure data at the mouth, as well as computed tomography (CT) images are obtained for each patient during normal breathing. The CT images are then processed to obtain the flow geometries needed for computational modeling. The trachea is separated from the lungs at the second level of bifurcation so that two separate physical geometries are obtained. A Meshless incompressible flow modeling routine is then used to predict the airflow through the trachea over a normal breathing cycle, where the inlet conditions are obtained from the measured data. The outlets of the trachea flow model are connected to the lung model and provide the link between the lung and trachea flow fields. The incompressible flow modeling is carried out by solving the Navier-Stokes equations using the approach previously as described by Kassab and Divo [4].

The lungs are modeled as poro-elastic media, where the flow field satisfies Darcy's Law and the elasticity field is solved using Navier's equation:

$$
\begin{gathered}
\phi \beta_{f} \frac{\partial p}{\partial t}=\nabla\left[\frac{\kappa}{\mu_{f}}(\nabla p)\right]-\frac{\partial}{\partial t}\left(\frac{\partial u_{i}}{\partial x_{i}}\right) \\
G \nabla^{2} \vec{u}+\frac{G}{1-2 \nu} \nabla(\nabla \cdot \vec{u})=\nabla p
\end{gathered}
$$

where $\vec{u}$ is the tissue deformation, $\nu$ is the constant tissue Poisson ratio, $\mu_{f}$ is the constant air viscosity, and $p$ is the pore pressure. The tissue properties in the above equations, porosity, $\phi$, permeability, $\kappa$, and shear modulus, $G$, are considered non-homogeneous as they can vary considerably throughout an individual's lung. The distribution of these properties for each patient are provided by a novel image processing technique as described by Santhanam et al. [2]. The overall coupling process is further illustrated using a simple 2D sketch in Figure 1. It should be noted that Eqn. (2) is given in steady-state form, while Eqn. (1) is 
transient. This is done as this is the approach utilized herein, as it is assumed that the lung deformation occurs at a much faster rate than the flow field changes within the porous lung tissue. Therefore, each time-step of the overall solution procedure requires the solution of the steady-state Navier equation, but much of the computational expense of this procedure is reduced since each time-step involves only a slight change in the deformation field solution.

\section{Meshless modeling technique}

The merits and benefits of Meshless Method solution approaches are well described in the literature, where it is generally noted that a reduction in the dependence of solution quality on the discretization is the most important achievement. Since this work relies on medical imaging data to provide the model geometries, it is difficult to guarantee that a smooth, clean geometry is obtained. This difficulty quickly leads one to assume that Meshless Methods are an excellent choice for use in this field of research, however, we have found that this is only true for certain formulations of Meshless Methods (the name Meshless Methods extends to include several distinct types of techniques). Furthermore, due to these geometric difficulties we have found that method robustness and stability are the key factors rather than accuracy in this field of numerical modeling. We have therefore adopted several different techniques, which are typically found within various Meshless Method approaches but rarely combined, into a single unified Meshless Method for the flow field modeling in this work. Specifically, this work utilizes radial basis function (RBF) interpolation, moving-least-squares (MLS) approximation, as well as finite differencing to create robust and efficient numerical solution schemes for both incompressible and poroelastic flows. Each of these techniques is applied in a localized manner to decrease computational effort and ensure the highest possible efficiency in the method. Some pertinent details regarding various Meshless-based methods as well each of the schemes mentioned are given in the remainder of this section, followed by an overview of how the various schemes are brought together to form a unified flow solution technique.

Typical Meshless Methods begin by assuming that any desired field, $\phi$, can be locally approximated by multiplying a set of basis functions, $\chi$, by a corresponding set of expansion coefficients, $\alpha$, as:

$$
\phi(x)=\sum_{j=1}^{N F} \alpha_{j} \chi_{j}(x)
$$

where $N F$ is a number of local expansion points (support domain) and $x$ refers to any number of coordinate directions. A critical component of these local Meshless techniques is the determination of a suitable set of basis functions that will accurately represent the field between the data points and provide appropriate estimations of the required field derivatives to facilitate the solution of the governing equation at hand. Many researchers, including the authors [4-7], have investigated this issue and several conclusions can be drawn from these previous 
works. For RBF methods, the Inverse Hardy Multiquadric [8] has repeatedly been noted as the best choice with regards to both stability and accuracy, while for MLS methods, standard monomial terms up to second order are typically found sufficient for most applications. Further details of these two approximation schemes are continued below.

Radial Basis Function interpolation has been the foundation of most Meshless Method formulations since the developments of Kansa over 20 years ago [9, 10]. Since those works, much more attention has been given to the specific details of the RBF schemes resulting in much gains in accuracy and stability of the techniques. Many researchers have reported such improved results by augmenting the RBFs with additional functions that are chosen specifically for the application at hand. Such augmentations are accomplished by updating the field approximation as:

$$
\phi(x)=\sum_{j=1}^{N F} \alpha_{j} \chi_{j}(x)+\sum_{k=1}^{N P} \alpha_{(k+N F)} P_{k}(x)
$$

where $P_{k}(x)$ refers to any desired set of basis functions and $N P$ refers to the number of extra basis functions used. Kassab and Divo [4], showed the importance of including constant and linear monomial terms, and Sarlar et al. [11] found much benefit to adding hyperbolic functions when solving heat transfer and other diffusion like equations. Despite the improvements gained by addition of these extra basis functions, much of the accuracy of the RBF techniques still depend heavily on the selection of a suitable "free parameter" that appears in the Hardy Multiquadric as:

$$
\chi\left(x_{i}\right)=\frac{1}{\sqrt{r_{j}\left(x_{i}\right)^{2}+c^{2}}}
$$

where $x_{i}$ is the point at which the field is being estimated, $r_{j}$ is the Euclidean distance from $x_{i}$ to point a point $x_{j}$ within the local influence zone, and $c$ is the free parameter. Earlier works by the authors $[4,12]$ have presented the details of a method to optimize the value of this free parameter for each and every interpolation point in the field. This optimization has proven to provide a very reliable and accurate RBF interpolation scheme when using the Hardy Multiquadric RBF. Once the basis functions have been selected, the task of converting any desired partial differential equation into an algebraic expression becomes quite straightforward, as each necessary differential operator may be applied directly to the basis functions and the resulting approximation equation may be solved by any standard type of explicit or implicit solution scheme.

Despite the excellent performance of RBF Meshless Methods and the relative ease of their implementation in many problems and application areas, they still suffer from three key deficiencies: convective derivatives are difficult to capture due to the radial symmetry of the RBFs; areas of locally Cartesian structure reduce to nearly the same results as standard finite differencing, but much more effort is expended in computing the required RBF weights; high gradient regions, such as shocks and boundary layers, can cause oscillations in the RBF interpolator, 
resulting in poor numerical instability. Therefore, other approximation schemes are necessary in some cases to alleviate these concerns.

Moving Least Squares approximations have gained a lot of attention in Meshless Methods in the area of global Meshless formulations [13-15], but have not seen much application in the area of localized approaches. However, our experience has found that MLS formulations provide much more stability when compared to RBF schemes, since much lower order approximation functions are utilized. Throughout our work we have found that quadratic MLS approximations are sufficiently accurate and very stable for a wide variety of application domains. For three-dimensional modeling, a quadratic MLS requires the following ten monomial terms:

$$
\chi(x)=\left\{\begin{array}{llllllllll}
1 & x & y & z & x y & y z & z x & x^{2} & y^{2} & z^{2}
\end{array}\right\}
$$

It should be noted that since the MLS approximations in this work are applied only over a small influence domain and are fully independent from the approximations in other domains, additional weighting functions are not needed. Also, it should be noted when using MLS approximations the local support domain must be much larger than that of typical RBF interpolations, and our implementations have been found most promising when it is ensured that at least 20 points with a minimum of four unique values in each coordinate direction are included (in other words, the support domain nodes must lie in at least 4 distinct planes when viewed from each coordinate direction). The typical disadvantage of MLS schemes comes from the fact that they are approximating and not interpolating functions, therefore applying boundary conditions can be a very complex process. However, we have found that by combining the MLS approximation approach into a generalized finite differencing-like scheme, coined Virtual Finite Differencing in our previous work [12,16], the application of both Dirichlet and Neumann type boundary conditions is straight-forward and obvious.

The above descriptions have been presented only with minor details as they are each well described in other works, however, what does not seem to have been undertaken by those working in the field of Meshless Methods is the combination of these techniques within a single computational implementation. Such a combination has been a major focal point of this research as the complex geometries present require much more stability than many other types of applications. We have attempted to optimize this combination through a rigorous testing and validation process.

Our recent works $[16,17]$ have shown that maintaining a Cartesian nodal structure throughout as much of the volume of the computational domain as possible allows classic finite differencing to be used in much of the domain (note that this is exactly equivalent to RBF Meshless methods for a 7-noded influence region as shown in previous works [12]), which significantly increases the computational efficiency. This approach of maintaining Cartesian nodal distributions has therefore been adopted in this work as well. This leaves only the boundary, near-boundary, and those interior regions where different discretization 
sizes come together where truly "Meshless" Methods must be used. For these interior nodes a nearly Cartesian structure still exists, which provides high quality local influence domains and allows RBF interpolation to be used without reservation. For the near boundary regions, the MLS Virtual Finite Differencing (MLS FD) approach is utilized, as stability is the major concern in this area. For the boundary itself, a combination approach exists. RBF interpolations are used directly on the boundary surface for the application of tangential boundary conditions, such as the traction conditions in elasticity applications. The MLS FD approach is then used for the normal direction boundary conditions or a pure finite difference is used when so-called "shadow nodes" are included in the computational domain (a thorough discussion of shadow nodes has been provided by the authors in several previous works $[4,12])$. Lastly, when boundary derivative approximations are needed, but are not the applied boundary conditions (for example when computing the dilatation on the boundary in fluid applications) standard differentiation of the MLS approximating functions are utilized. The combination of schemes has been found to significantly improve the numerical stability of our Meshless Methods and further reduce the dependence of the solution on the quality of the underlying nodal distribution. It should be noted however, that as with any computational modeling scheme, accuracy is still a function of the local nodal spacing, therefore our implementations all include some type of automatic, solution based nodal refinement strategy, so that accuracy and "discretization convergence" is achieved for all our solutions.

\section{Current results}

The overall solution approach to tracheobronchial flow modeling utilized in this work has been verified for simulated two-dimensional configurations in our previous work [18]. These verifications provided some confidence to the soundness of the coupling schemes and the ability of the Meshless methods to capture the necessary flow physics. The extension to 3D and application to real patient lung geometries necessitated the solution approach updates outlined above. The approach is now capable of predicting lung tissue deformations for real patients and is currently undergoing verification in the clinic for real cancer patients.
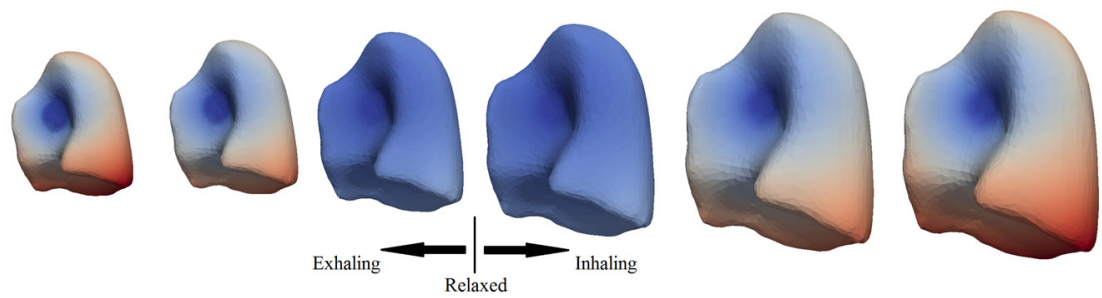

Figure 2: Example patient lung deformation results throughout breathing cycle (sized according to deformation and colored by deformation magnitude). 
The lung deformation fields throughout time are shown below for one particular patient. Figure 2 shows the entire right lung and Figure 3 tracks the deformation history for a single point on the outer lung surface. An image reconstruction process is currently being completed that will allow the comparison of these deformation fields with the real patient lung motions obtained from time dependent (4D) CT scans. This process will enable the elasticity field boundary conditions to be properly varied with deformation, so that the computational models can more accurately match the real patient lung deformations. This next phase of research is currently underway for several patients.

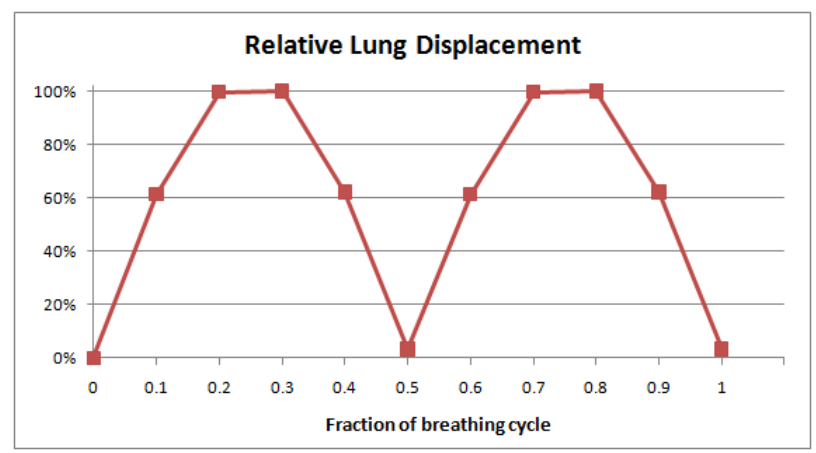

Figure 3: Relative displacement of a landmark point on the outer lung surface throughout the breathing cycle.

\section{Conclusions}

We have presented a new generalized Meshless Method approach that allows the tailoring of a Meshless method to achieve the most important goals for any application by selecting schemes with the most desirable properties for each different type of circumstance that is encountered within the solution process. It is suggested that: finite differencing be applied to the bulk of the interior to save effort on the pre-processing stages; RBF interpolation be applied along the boundary surfaces and within the volume for areas with suitable quality nodal discretizations; and the MLS approximations be used for near-boundary derivative estimations and for the application of generalized finite differencing in low quality distribution regions. Furthermore, it is our recommendation from this research that others explore such generalized approaches to improve other Meshless techniques in any areas of concern as well.

\section{Acknowledgement}

We would like to acknowledge the funding received in support of this project from the James and Esther King Biomedical Research Program. 


\section{References}

[1] Stevens C, Munden R, Forster K, Kelly J, Zhongxing L, Starkschell G, Tucker S, Komaki R. Respiratory-driven lung tumor motions is independent of tumor size, tumor location, and pulmonary function. International Journal of Radiation Oncology 2001; 51:62-68.

[2] Santhanam A, Willoughby T, Shah A, Meeks S, Rolland J, Kupelian P. Realtime simulation of $4 \mathrm{~d}$ lung tumor radiotherapy using a breathing model. MICCAI 2008; 2:710-717.

[3] Kowalczyk P. Mechanical model of lung parenchyma as a two-phase porous media. Journal of Transport in Porous Media 1993; 11:281-295.

[4] Kassab A, Divo E. An efficient localized radial basis function meshless method for fluid flow and conjugate heat transfer. ASME Journal of Heat Transfer 2007; 129:179-183.

[5] Divo E, Kassab A. An efficient localized rbf meshless method applied to fluid flow and conjugate heat transfer. Proceedings of ASME IMECE, 2005.

[6] Sarler B, Tran-Cong T, Chen C. Meshfree direct and indirect local radial basis function collocation formulations for transport phenomena. Boundary Elements XVII, Kassab A, Brebbia C, Divo E (eds.), WIT Press, 2005; $417-$ 428.

[7] Shu C, Ding H, Yeo K. Local radial basis function-based differential quadrature method and its application to solve two-dimensional incompressible navier-stokes equations. Computer Methods in Applied Mechanics and Engineering 2003; 192:941-954.

[8] Hardy RL. Multiquadric equations of topography and other irregular surfaces. Journal of Geophysical Research 1971; 76:1905-1915.

[9] Kansa EJ. Multiquadrics: A scattered data approximation scheme with applications to computational fluid dynamics ii-solutions to parabolic, hyperbolic and elliptic partial differential equations. Computers and Mathematics with Applications 1990; 19:147-161.

[10] Kansa EJ. Multiquadrics: A scattered data approximation scheme with applications to computational fluid dynamics i-surface approximations and partial derivative estimates. Computers and Mathematics with Applications 1990; 19:127-145.

[11] Sarler B, Vertnik R. Local explicit radial basis function collocation method for diffusion problems. Computers and Mathematics with Applications 2006; 51:1269-1282.

[12] Gerace S. An interactive framework for meshless methods analysis in computational mechanics and thermofluids. Master's Thesis, University of Central Florida 2007.

[13] Belytschko T, Lu YY, Gu L. Element-free galerkin methods. International Journal of Numerical Methods 1994; 37:229-256.

[14] Belytschko T, Krongauz Y, Fleming M, Organ D, Liu WKS. Smoothing and accelerated computations in the element free galerkin method. Journal of Computational and Applied Mathematics 1996; 74(1):111-126. 
[15] Atluri SN, Zhu T. A new meshless local petrov-galerkin (mlpg) approach in computational mechanics. Computational Mechanics 1998; 22:117-127.

[16] Gerace S, Erhart K, Divo E, Kassab A. Generalized finite difference meshless method in computational mechanics and thermofluids. Proceedings of the Int. Conf. on Comp. Methods for Coupled Problems in Science and Engineering (COUPLED PROBLEMS 2009), 2009.

[17] Erhart K, Gerace S, Divo E, Kassab A. Turbulent compressible flow analysis with meshless methods. Proceedings of the Int. Conf. on Comp. Methods for Coupled Problems in Science and Engineering (COUPLED PROBLEMS 2009), 2009.

[18] Huayamave V, Vidal A, Divo E, Kassab A, Santhanam A, Kupelian P. A meshless approach to solve the fluid poro-elastic interaction problem between the tracheo-bronchial tree and the lungs. Proceedings of the Int. Conf. on Comp. Methods for Coupled Problems in Science and Engineering (COUPLED PROBLEMS 2009), 2009. 